\title{
Principais Manifestações Neurológicas decorrentes do COVID-19: uma revisão integrativa
}

\author{
Main Neurological Manifestations arising from COVID-19: an integrative review \\ Principales Manifestaciones Neurológicas derivadas del COVID-19: una revisión integradora
}

\begin{abstract}
RESUMO
Objetivo: Identificar as principais manifestações neurológicas e suas implicações no quadro clínico do paciente bem como a fisiopatologia do SAR-CoV-2 no sistema nervoso central. Métodos: Uma revisão de literatura integrativa foi conduzida com base em 14 artigos extraídos da base de dados PUBMED e LILACS. Resultados: Foi constatado que cefaleia, vertigem, disfunções gustativas e olfatórias e AVC são os sintomas mais prevalentes. Conclusão: A fisiopatologia do envolvimento neurológico pelo COVID-19 permanece inconclusiva e conhecer a sintomatologia neurológica associada aos quadros mais graves e severos pode auxiliar em intervenções precoces e na melhora de prognósticos.
\end{abstract}

DESCRITORES: Sistema Nervoso; Manifestações Neurológicas; Infecções por Coronavirus.

\section{ABSTRACT}

Objective: To identify the main neurological manifestations and their implications in the patient's clinical condition as well as the pathophysiology of SAR-CoV-2 in the central nervous system. Methods: An integrative literature review was conducted based on 14 articles extracted from the PUBMED and LILACS database. Results: It was found that headache, vertigo, taste and olfactory disorders and stroke are the most prevalent symptoms. Conclusion: The pathophysiology of neurological involvement by COVID-19 remains inconclusive and knowing the neurological symptoms associated with the most severe conditions can assist in early interventions and in improving prognosis.

DESCRIPTORS: Nervous System; Neurologic Manifestations; Coronavirus Infections.

\section{RESUMEN}

Objetivo: Identificar las principales manifestaciones neurológicas y sus implicaciones en la situación clínica del paciente, así como la fisiopatología del SAR-CoV-2 en el sistema nervioso central. Métodos: Se realizó una revisión integradora de la literatura a partir de 14 artículos extraídos de la base de datos PUBMED y LILACS. Resultados: Se encontró que la cefalea, el vértigo, los trastornos del gusto y olfativos y el ictus son los síntomas más prevalentes. Conclusión: La fisiopatología de la afectación neurológica por COVID-19 sigue sin ser concluyente y conocer los síntomas neurológicos asociados con las condiciones más graves puede ayudar en las intervenciones tempranas y en la mejora del pronóstico.

DESCRIPTORES: Sistema Nervioso; Manifestaciones Neurológicas; Infecciones por Coronavirus.

RECEBIDO EM: 15/10/2020 APROVADO EM: 06/11/2020

\section{Lília Tereza Diniz Nunes}

Acadêmica do curso de Medicina da FAHESA/ITPAC Palmas, Graduada em Moda pelo Centro Universitário das Faculdades Metropolitanas Unidas (2010). Pós-Graduanda em Docência do Ensino Superior pelo ITOP-Palmas. Mestre em Design pela Universidade Anhembi Morumbi.

ORCID: 0000-0002-8571-3039

\section{Luiz Eduardo Cunha Felipe}

Acadêmico do curso de Medicina da FAHESA/ITPAC Palmas, Ensino Superior Incompleto

ORCID: 0000-0001-6906-7201 


\section{artigo}

Nunes, L.T.D.; Felipe, L.E.C.; Gonçalves, I.M.; Alves, O.N.;

Principais Manifestações Neurológicas decorrentes do COVID-19: uma revisão integrativa

\section{Itamar Magalhães Gonçalves}

Graduado em Medicina pela Universidade Federal de Uberlândia, Residência Médica em Cirurgia Geral e Urologia pela Universidade Federal de Uberlândia e Mestre em Ensino em Ciências da Saúde pela Universidade Federal de São Paulo. Já atuou como professor do curso de Medicina da Universidade Federal do Tocantins e Coordenador do Programa Telessaúde Tocantins Redes. Atualmente, exerce a função de Perito Médico Legista da Secretaria de Segurança Pública do Estado do Tocantins e atua como Professor do Curso de Medicina do Instituto Tocantinense Presidente Antônio Carlos - ITPAC Palmas. Interessa-se por Ensino em Ciências da Saúde, Tecnologias de Informação e Comunicação, Exercícios de Simulação e Habilidades Médicas. ORCID: 0000-0003-2276-6288

\section{Oscar Nunes Alves}

Graduado em Medicina pela Universidade Federal do Espírito Santo, título de Neurocirurgião pela Sociedade Brasileira de Neurocirurgia. Já atuou como Professor do Curso de Medicina do Instituto Tocantinense Presidente Antônio Carlos - ITPAC Porto Nacional e como Chefe do Serviço de Neurocirurgia do Hospital Geral de Palmas. Atualmente concursado como Neurocirurgião do Hospital Geral de Palmas, atendendo também a hospitais privados e é sócio proprietário do Instituto de Neurociências do Tocantins.

ORCID: 0000-0002-6443-6853

\section{INTRODUÇÃO}

0 novo coronavírus e a pandemia gerada por ele mudou drasticamente o mundo e o sistema de saúde. Alguns estudos feitos logo no início da pandemia demonstraram sintomatologia de predomínio respiratório incluindo febre, tosse, dispneia e fadiga ${ }^{1}$. Com o desenvolvimento da doença e novos casos em todo o mundo foi possível identificar o tropismo do vírus também por células neurais através de mecanismos que ainda estão sendo estudados.

"Até o final da Semana Epidemiológica (SE) 35 de 2020, no dia 29 de agosto, foram confirmados 24.761 .119 casos de COVID-19 no mundo. Os Estados Unidos foram o país com o maior número de casos acumulados (5.917.439), seguido pelo Brasil (3.846.153)"2

Essa pesquisa visa analisar por meio de levantamento de dados publicados em 10 artigos levantados na base de dados PUBMED no período de tempo de dezembro de 2019 a agosto de 2020 quais são os principais sintomas neurológicos apresentados por pacientes acometidos pelo SARS-CoV-2. Multiplicidade de sintomas neurológicos foram encontrados ao analisar os artigos escolhidos desde sintomas inespecíficos como cefaleia, náuseas e vômitos a sintomas específicos como AVC, Síndrome de Guillain Barré e doenças de nervos periféricos.

Várias pesquisas têm se dedicado a entender como o SARS-CoV-2 acessa o cére- bro causando as manifestações neurológicas apresentadas pelos pacientes, Whittaker et $\mathrm{al}^{3}$ propõe duas teorias, a primeira seria através de disseminação vascular sistêmica e a segunda e mais local através da lâmina cribriforme do osso etmoide.

Algo é certo, o neurotropismo do vírus é familiar como o apresentado pelo SAR$s-\mathrm{CoV}$ que penetra a célula via uma proteína chamada ECA2, expressa no epitélio das vias aéreas superiores e inferiores, bem como, no epitélio endotelial do sistema nervoso central.

SARS-CoV-2 usa o receptor da enzima conversora de angiotensina 2 (ECA2) para entrar nas células hospedeiras, o mesmo que a infecção por SARS-CoV fez em 2003. O mecanismo patológico que COVID-19 tem no sistema nervoso pode acontecer por várias vias, incluindo a via hematogênica, via neural retrógrada, hipóxia, lesão imune e enzima ECA2. O vírus entrando na circulação sanguinea pode fazer com que o sistema imunológico produza citocinas como resposta fisiológica; o aumento da produção de citocinas pode causar um aumento da permeabilidade $d a$ barreira hematoencefálica, $f a-$ cilitando assim a entrada do vírus no SNC. Isso pode explicar porque os pacientes com COVID-19 mais graves podem ter Sindrome de tempestade de citocinas'.
Quanto a progressão do vírus no sistema nervoso alguns estudos têm postulado uma disseminação neuronal do coronavírus em que o vírus infecta um neurônio periférico e através da maquinaria de transporte ativo, terminal sináptico e transporte retrógrado é levado do corpo celular neuronal para áreas remotas do cérebro. Este mecanismo de transferência trans-sináptica é apoiado por estudos envolvendo a cepa $67 \mathrm{~N}$ hemaglutinante do vírus da encefalomielite, a primeira cepa $\mathrm{CoV}$ encontrada a invadir o cérebro5.

A evidência adjuvante para envolvimento neurológico em COVID-19 inclui exame de LCR, eletrofisiologia, e achados radiológicos. A mudança característica no LCR após COVID-19 é o ligeiro aumento nas contagens de células e nos níveis de proteínas, especialmente imunoglobulinas, o que sugere estado inflamatório ou infeccioso ${ }^{6}$.

Nessa revisão integrativa iremos apresentar os principais sintomas neurológicos envolvidos no curso da doença, sua fisiopatologia e aprofundar nos quatro sintomas mais prevalentes em pacientes com COVID-19.

\section{MÉTODO}

Para a realização deste estudo, optou-se pela revisão integrativa da literatura, fundamentada nos estudos de Ganong 7 , Broome ${ }^{8}$ e Whittemore e Knalf 9

Para a elaboração da presente revisão 
integrativa as seguintes etapas foram percorridas: estabelecimento da hipótese e objetivos da revisão integrativa; estabelecimento de critérios de inclusão e exclusão de artigos (seleção da amostra); definição das informações a serem extraídas dos artigos selecionados; análise dos resultados; discussão e apresentação dos resultados e a última etapa consistiu na apresentação da revisão.

Para guiar a revisão integrativa, formulou-se a seguinte questão: quais são os principais sintomas neurológicos decorrentes da infecção por coronavírus?

Para a seleção dos artigos foram utilizadas duas bases de dados o PubMed e a Lilacs, procurando ampliar o âmbito da pesquisa e evitar vieses.

Os critérios de inclusão inicialmente definidos para a revisão foram: artigos em português, inglês e espanhol, com os resumos disponíveis nas bases de dados selecionadas, publicados no período compreendido entre dezembro de 2019 e agosto de 2020, artigos publicados cuja metodologia adotada permitisse obter dados concisos assim excluiu-se revisões de literatura, carta ao editor, artigos de opinião e artigos sem dados clínicos ou fora da temática abordada.

Foram utilizados os descritores em saúde Nervous System, Coronavirus Infections e o operador boleano AND. Inicialmente foram encontrados 638 resultados que foram refinados com os seguintes filtros: " $\mathrm{Cli}$ nical Trial”, " Meta-Analysis", "Randomized Controlled Trial e Sistematic Review. Após a leitura na íntegra de seus conteúdos optou-se por utilizar 14 artigos que atenderam aos critérios elencados anteriormente.

Para avaliação dos dados, elaborou-se

\section{Figura 1: Fluxograma seleção de artigos}

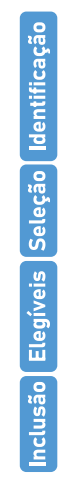

\section{PUBMED e LILACS}

Keywords: Nervous system and COVID-19
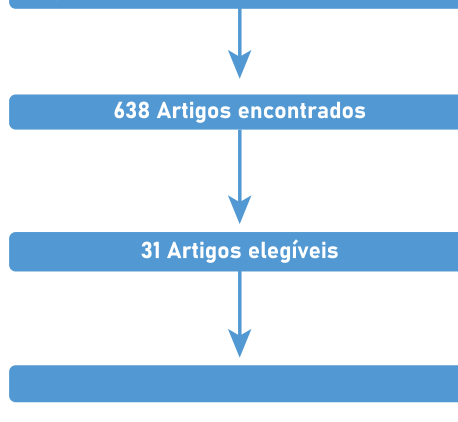

Fonte: Autoria própria (2020) um instrumento de coleta das informações visando responder à questão norteadora desta revisão. A análise e interpretação dos dados foram realizadas de forma organizada e sintetizada por meio da elaboração de um quadro sinóptico que compreendeu os seguintes itens: identificação do estudo; objetivos, ano e periódico de publicação; delineamento do estudo; temática; e, principais resultados e recomendações. Os artigos selecionados foram analisados na íntegra e agrupados por áreas temáticas. A validação dos dados deu-se através do instrumento PRISMA verificado por dois pesquisadores profissionais da área da saúde.

A apresentação dos resultados e discussão dos dados obtidos foi feita de forma descritiva, através de tabelas e gráficos possibilitando ao leitor a avaliação da aplicabilidade da revisão integrativa elaborada, de forma a atingir o objetivo desse método.

\section{RESULTADOS}

Foi realizada uma tabulação dos sintomas neurológicos encontrados nas pesquisas escolhidas e dividiu-se esses sintomas em primeiramente pelos seus artigos visando conhecer as diversas manifestações neurológicas conforme tabela a seguir. Posteriormente foi feito um gráfico para conhecer a prevalência dos 10 sintomas mais descritos na tabela 1 e desses 4 foram destacados para serem analisados em categorias temáticas devido a sua alta incidência.

Pode-se ver uma gama ampla de sintoma-

\section{Tabela 1: Sintomas Neurológicos}

Autor

Tipo de Estudo

WHITTAKER, ANSON e HARKY, 2020

Revisão Sistemática

ASADI-POOYA, SIMA$\mathrm{NI}, 2020$

Revisão Sistemática

WANG et. al, 2020

Revisão Sistemática com Meta- Análise

\section{Sintomas Neurológicos Encontrados}

Ataxia, Síndrome de Guilan Barré, Cefaleia, Vertigem, Convulsões, Encefalopatia, Desorientação, Anosmia, Disfunção olfatórias e gustativas, Agnosia, Alucinações, AVC Isquêmico, Ataxia, Hiporeflexia, Confusão mental, EEG alterado, Perda da consciência, Redução do Nível de Consciência, Status Epiléptico, Fraqueza bilateral dos membros inferiores e dormência, Olhar sustentado para cima, Endurecimento bilateral da perna

Cefaleia, Vertigem, Redução do nível de consciência, Doenças cerebrovasculares agudas, Ataxia, Convulsões, AVC isquêmico, Trombose do seio venoso cerebral, Hemorragia cerebral, Confusão mental

Consciência prejudicada, Doença cerebrovascular aguda, Sinais de liberação do trato corticoespinhal, Ataxia, Vertigem, Disfunção gustativa e olfatória, Disfunção visual e dores nos nervos. 


\section{artigo}

Nunes, L.T.D.; Felipe, L.E.C.; Gonçalves, I.M.; Alves, O.N.

Principais Manifestações Neurológicas decorrentes do COVID-19: uma revisão integrativa

NEPAL et al, 2020

MONTALVAN et al, 2020

CHEN et al, 2020

FÁTIMA et al, 2020

Revisão Sistemática

ROMOLl et al, 2020

Revisão Sistemática

WILSON e JACK, 2020

Revisão Sistemática

Revisão Sistemática com Meta-Análise

PINZON et al, 2020

MSIGWA et al, 2020

Revisão Sistemática

ROMÁN et al, 2020

Revisão Sistemática

KREMER et al, 2020

Estudo retrospectivo

ROMERO-SANCHEZ et al, 2020
Cefaleia, Encefalopatia, AVC isquêmico, Hemorragia Intracerebral, Encefalite, EncefaIomielite, Mielite Aguda, Disfunção gustatória e olfativa, Paralisia de Bell, Síndrome de Guillain Barré, Mialgia, Miosite, Rabidomiólise

\section{Encefalite, Polineuropatia, Miopatia, AVC, Esclerose Múltipla}

Cefaleia, Vertigem, Disfunções gustativas e olfatórias, redução do nivel de consciência, Doenças cerebrovasculares agudas, Convulsões, Encefalite, Meningite, Síndrome de Guillain Barré, Síndrome de Miller Fisher e Paralisia do Nervo Oculomotor

AVC Isquêmico, AVC Hemorrágico, Trombose.

Disfunção gustativa e olfatória, Disfunção visual, Cefaleia, Mudanças de humor, Febre, Convulsões, Meningite, Encefalopatia, Oftalmoparesia internuclear direita, Neuropatias craniais multiplas, Confusão mental, Agitação, Sinais piramidais, Sindrome disexecutiva, Vertigem, Dor em nervo, Manifestações musculo esqueleticas, Coma, Rigidez de pescoço, Fraqueza simetrica e distal de membros moderada, Perda de reflexos, Quadriplegia aguda simetrica progessiva, Tetraplegia de arreflexia flácida, Paralisia, Diplegia facial, Parestesia de membro, Fraqueza simetrica, Arreflexia, Diminuição da sensação termo-dolorosa distal, Parestesia flacida, Incontinência urinaria e fecal

Cefaleia, Vertigem, Redução do nivel de consciência, Hipoalgesia, Disfunções olfatórias ou anosmia e Neuralgia, AVC agudo, epilepsia e ataxia

Cefaleia, Vertigem, Disfunções olfatória e gustativas, Disfunções visuais, Fraqueza muscular, Doença cerebrovascular aguda, Convulsões, Ataxia, Neuralgia, AVC, Miopatia, Polineuropatia, Rabidomiólise, Convulsões, Redução do nível de consciência e Encefalopatia

Estado de mal epiléptico, convulsão com duração de 5 a 30 minutos ou mais sem anulação automática, convulsões reaparecendo repetidamente sem restauração total da consciência; Confusão, Convulsão generalizada; encefalite e meningite associadas a confusão e perda de consciência e sinais de irritação meníngea; AVC agudo; Infartos cerebrais de grandes vasos; trombose e estado hipercoagulável; encefalomielite disseminada aguda; Estado mental alterado, com ataxia e déficits motores focais; coma, Distúrbios neuromusculares; Polineuropatia critica, Miopatia, Rabdomiólise; Neuropatia olfatória; Anosmia e Ageusia, paralisia do nervo abducente bilateral, arreflexia e dissociação albuminocitológica,

Doença cerebrovascular; Hipoperfusão frontotemporal (agitação; sinais do trato corticoespinhal, como reflexos tendinosos acelerados, clônus do tornozelo e respostas plantares extensoras bilaterais; delirium; e hipertermia com febre, encefalopatia); tromboses arteriais e venosas, hemorragia subaracnóide e coagulopatias; Encefalopatia necrosante hemorrágica aguda; Encefalopatia, meningite, encefalite, convulsões;

Síndrome de Guillain-Barré; Síndrome de Miller Fisher, Polineurite cranial; Mielite; Miastenia Gravis; Miopatias, Cefaleia, Disfunções Gustativas e Olfatórias, AVC agudo ( isquêmico e hemorrágico)

AVC isquêmico, Hemiplegia esquerda; Sinais do trato piramidal bilateral; Fraqueza do lado esquerdo, Desatenção sensorial do lado esquerdo; Disartria; Afasia, Hemiplegia direita, Fraqueza do lado direito, Queda facial direita, Hemianopsia homônima esquerda, Meningoencefalite, Confusão, Agitação, Vigilia patológica quando a sedação foi interrompida, Consciência prejudicada, Cefaleia, Vertigens, Ataxia, Distúrbios do movimento.

Mialgias; Cefaleia; Vertigem; Síncope; Anosmia; Disgeusia; Desordens de consciência; Sonolência; Estupor; Coma; Bradicipsíquia, Desorientação; Síndrome confusional aguda; Convulsões; Disautonomia; Lesões musculares (Hipercalcemia, Rabdomiólise, Miopatia); AVC isquêmico; Hemorragia intracraniana; Distúrbios do movimento (hipercinético); Encefalite; Neurite óptica; Sintomas neuropsiquiátricos (ansiedade, depressão, insônia, psicose) 
tologia neurológica, porém alguns sintomas se destacaram, dez deles estiveram presentes em ao menos três das pesquisas escolhidas e foram distribuídas no Gráfico 1.

Dentre essas destacam-se quatro Cefaleia, Vertigem, Disfunções Gustativas e Olfatórias e AVC devido à sua alta prevalência entre os artigos estudados (citadas em pelo menos 10 dos 14 artigos estudados) foram analisados separadamente em categorias temáticas de acordo com a abordagem quantitativa apresentadas nas pesquisas estudadas e serão abordadas a seguir.

\subsection{CEFALEIA:}

Dos 31 artigos estudados por Whittaker et $\mathrm{al}^{3} 12$ deles apresentavam casos onde a cefaleia foi relatada como sendo um dos sintomas prevalentes entre os pacientes, em um dos estudos retrospectivos analisados a prevalência chegou a $35 \%$ sendo descrita como um dos sintomas neurológicos mais comuns. Asadi-Pooya10, demonstra achados similares ao de Whittaker ${ }^{3}$ ao mostrar a prevalência da cefaleia variando entre 6 a $13 \%$ e dos 6 artigos estudados houve a presença da cefaleia como manifestação neurológica em 5 deles. Segundo Nepal et al4, oito estudos retrospectivos reportaram pacientes com COVID-19 apresentando cefaleia, o que representa uma média de 19,88\%.

A pesquisa de Chen et al ${ }^{11}$ relata importantes dados ao apontar que a cefaleia foi encontrada em 51 estudos envolvendo 16.446 mil pacientes com COVID-19,

\section{Gráfico 1: Principais Manifestações Neurológicas}

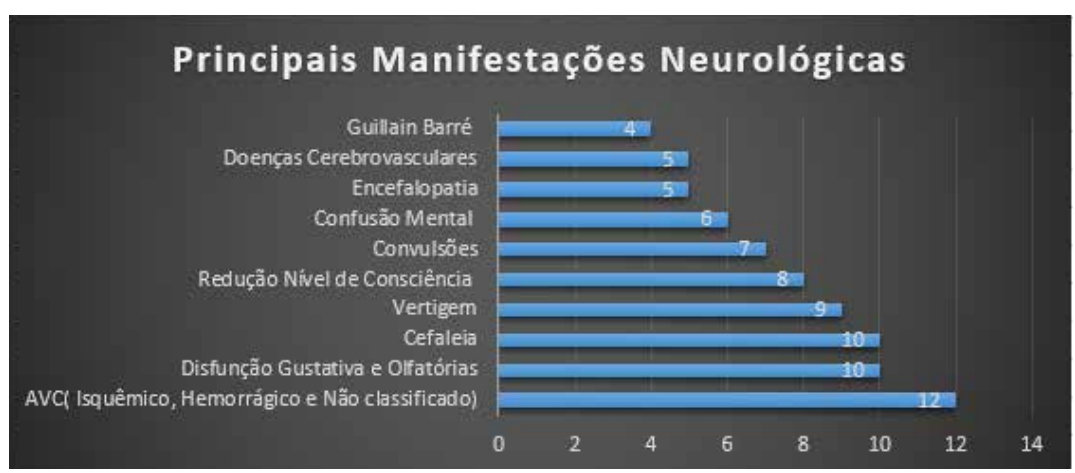

Fonte: Autoria Própria,2020

desses, a cefaleia foi reportada em $20,1 \%$ da população estudada e também relata a presença da cefaleia associada a vertigem em mais 8 casos que envolveram 654 pacientes com COVID-19.

Romoli et a ${ }^{12}$ descreveu a presença de cefaleia em 4 dos 27 artigos analisados. Wilson e Jack1, encontraram a cefaleia dentre os sintomas neurológicos mais comuns com prevalência de 11 a $13 \%$. Pinzon et a ${ }^{13}$ relatou a presença de cefaleia em 21 estudos (em um universo de 33) sendo o sintoma mais comum apresentado logo após a mialgia com prevalência de $34 \%$.

Román et al14, 2020 descreve que em um estudo coorte de 262 casos confirmados em hospitais de Beijing hospitais 6.5\% tinham cefaleia comparado com 6 a $8 \%$ em Wuhan.

Kremer et $\mathrm{al}^{15}, 2020$ encontrou a cefaleia no universo dos sintomas neurológicos mais comuns decorrentes do COVID-19, dentre os 64 pacientes estudados 10 apresentarem este sintoma o que corresponde a $16 \%$.

Romero-Sanchez et a ${ }^{16}, 2020$ classificou a cefaleia como sintoma neurológico não específico e junto com a mialgia foi o sintoma mais comum encontrado, em um universo de 841 pacientes, 119 deles apresentaram cefaleia o que representa $14,1 \%$ de prevalência corroborando estatísticas apresentadas por autores acimas demonstrados.

\subsection{VERTIGEM:}


pacientes com quadro de COVID-19 leve ou moderado quando comparado à quadros severos e graves.

Romoli et al $^{12}$ encontrou taxas ainda mais altas de disfunções gustativas e olfatórias variando de $85,6 \%$ a $88 \%$. Pinzon et a ${ }^{13}$ por sua vez relata apenas um estudo com prevalência de $5 \%$. Wilson e Jack ${ }^{1}$ também encontraram prevalência de 5\% para casos de anosmia e hiposmia.

Msigwa et $\mathrm{al}^{17}, 2020$ relata que anosmia e ageusia ocorrem em $>80 \%$ entre os casos de COVID-19 seja como uma primeira apresentação ou como o único sinal neurológico em casos leves.

Román et $\mathrm{al}^{14}$, 2020 demonstrou encontrar uma grande diferença entre os quadros de anosmia e disgeusia nos estudos feitos na China e na Europa, enquanto na China na cidade de Wuhan este sintoma esteve prevalente em 5,1\% dos casos estudados em 3 hospitais na Europa houve uma prevalência de 85,6\% em 12 hospitais, o mesmo padrão ocorre para a disgeusia que em Wuhan apresentava uma prevalência de 5,6\% em 3 hospitais e na Europa 88\% em 12 hospitais.

Romero- Sanchez et $\mathrm{al}^{16}$, 2020 relata percentuais mais similares aos chineses em sua pesquisa com 841 casos 41 deles apresentavam anosmia e 52 disgeusia o que corresponde a $4.9 \%$ e $6.2 \%$ respectivamente.

\subsection{ACIDENTE VASCULAR CEREBRAL}

Whittaker et $\mathrm{al}^{3}$, aponta a presença de 6 artigos com relato de AVC dentre os 31 estudados e em um dos estudos 5 pacientes menores de 50 anos incluindo dois que não tinham histórico médico pregresso apresentaram o quadro. Fatima et a ${ }^{18}$ revisou 39 pacientes dos quais 36 apresentaram AVC isquêmico $(92,3 \%)$ e 2 pacientes apresentaram AVC hemorrágico (5,1\%).

Asadi-Pooya et $\mathrm{a}^{10}$ encontrou percentuais mais baixos de AVC isquêmico em um de seus estudos com 221 pacientes 5\% deles apresentaram tal evento. Nepal et a ${ }^{4}$ apresentou resultados de estudos variados sobre AVC isquêmicos com prevalência de 2,8 a $5 \%$ de eventos isquêmicos, prevalência semelhante à apresentada por Wilson e Jack $^{1}$ de $3 \%$ para eventos cerebrovasculares agudos.

\section{Os sintomas do}

sistema nervoso

central (vertigens,

ataxia, cefaleia, AVC,

etc) foram mais

comuns do que os

do sistema nervoso

periférico. Os

sintomas inespecíficos

vertigem, cefaleia e

disfunções gustativas

e olfatórias apesar

de estarem presentes

em outros quadros

virais se tornaram

bem característicos

do quadro

sintomatológico
inicial do
COVID-19...

Segundo Montalvan et $\mathrm{al}^{5}$ AVC são complicações incomuns de infecções virais do sistema nervoso central, doenças de grandes vasos foram reportadas em 5 de 206 pacientes de COVID-19 além de terem sido relatadas em um estudo de uma série de casos 14 AVC em 214 pacientes com COVID-19. ${ }^{4}$

Román et $\mathrm{al}^{14}$, 2020 se aproxima dos autores ao relatar uma incidência de 5\% de AVC agudo incluindo isquêmico e hemorrágicos em séries de casos estudados na China.

Kremer et $\mathrm{a}^{15}, 2020$ promoveu um estudo com pacientes que tiveram AVC isquêmico devido ao COVID e encontrou 17 pacientes dos 64 estudados o que representa $26 \%$ dado que difere bastante dos encontrados por Romero-Sanchez ${ }^{16}$ ao relatar 11 casos dentre os 841 estudados levando a uma prevalência de $1,3 \%$

\section{DISCUSSÃO}

Os sintomas do sistema nervoso central (vertigens, ataxia, cefaleia, AVC, etc) foram mais comuns do que os do sistema nervoso periférico. Os sintomas inespecíficos vertigem, cefaleia e disfunções gustativas e olfatórias apesar de estarem presentes em outros quadros virais se tornaram bem característicos do quadro sintomatológico inicial do COVID-19, sugerindo níveis de envolvimento do sistema nervoso central.

Os acidentes vasculares cerebrais dos tipos isquêmicos e hemorrágicos foram descritos em 12 dos 14 artigos estudados, as disfunções gustativas e olfatórias em 10 de 14 , seguidos da cefaleia e em seguida vieram as vertigens.

A anosmia, hiposmia,hipogeusia, e disgeusia são sintomas muito comuns entre pacientes COVID-19 em todo o mundo chegando a índices de $88 \%{ }^{12}$. em geral estão associadas, porém quando isoladas a disfunção olfatória tem predomínio e tem sido sugerido que principalmente a anosmia poderia indicar o potencial neurotrópico do coronavírus para alcançar o cérebro através do nervo olfatório ${ }^{14}$.

Quando analisamos os dados apresentados sobre cefaleia nos deparamos com dois extremos, Whittaker et $\mathrm{al}^{3}, 2020$ relatou $35 \%$ de cefaleia nos pacientes estudados e Román et $\mathrm{al}^{14} 6,5 \%$ isso pode se dever ao fato de que muitas vezes esse sin- 
toma não é facilmente ligado como uma manifestação neurológica sendo tratado como sintoma geral e mais comum à infecções virais que relacionadas à um quadro clínico típico do COVID, porém o "início precoce da cefaleia e a diminuição da capacidade de resposta são indicadores de envolvimento neurológico potencial em pacientes COVID-19”14.

Ressaltamos assim como os autores que essas manifestações neurológicas inespecíficas que comumente aparecem precocemente na doença podem vir a ser importante preditoras de uma futura deterioração do quadro clínico ${ }^{3}$.

Os acidentes vasculares cerebrais são devido à natureza trombo-inflamatória do SARS-Cov-2 que predispóe os pacientes à um estado de hipercoagulabilidade devido à uma produção aumentada de fatores pró-coagulação e dano ao endotélio capilar o que resulta numa desregulação de suas propriedades anti-trombóticas ${ }^{3}$ - apesar de Whittaker et $\mathrm{al}^{3} \mathrm{em}$ sua pesquisa ter encontrado o tipo hemorrágico de AVC como mais prevalente (92\%) - aliado à isso está o fato que ambas as doenças apresentam fatores de risco similares predispondo a doença dos grandes vasos 5 .

O COVID-19 pode, portanto, aumentar o risco de tromboembolismo venoso e arterial associada a inflamação, hipóxia, e coagulação intravascular difusa ${ }^{14}$ sendo mais grave em pacientes idosos, que apresentem fatores de riscos em quadros mais graves da doença.

\section{CONCLUSÃO}

A completa compreensão dos mecanismos fisiopatológicos de invasão neural do SARS-CoV-2 permanece um desafio à medicina, o crescente número de achados sintomatológicos neurológicos dos mais variados tipos levanta a questão de que este vírus poderia ser um novo tipo de neuropatógeno subdiagnosticado. Através desta pesquisa foi possível rever os principais sintomas neurológicos e se aprofundar nos principais tipos, conhecimento este que pode ser um preditor de intervenções precoces, de melhor entendimento da atuação do vírus e consequentemente de alcance de melhores prognósticos nos pacientes afetados.

\section{REFERÊNCIAS}

1. Wilson MP, Jack AS. Coronavirus disease 2019 (COVID-19) in neurology and neurosurgery: A scoping review of the early literature. Clin Neurol Neurosurg. 2020 Jun;193:105866.

2. Ministério da Saúde, Secretaria de Vigilância em Saúde. BOLETIM EPIDEMIOLÓGICO ESPECIAL 29 Doença pelo Coronavírus COVID-19. 2020 Set 02:ISSN 9352-7864

3. Whittaker A, Anson M, Harky A. Neurological Manifestations of COVID-19: A systematic review and current update. Acta Neurol Scand. 2020 Jul;142(1):14-22.

4. Nepal G, Rehrig JH, Shrestha GS, Shing YK, Yadav JK, Ojha R, et al. Neurological manifestations of COVID-19: a systematic review. Crit Care. 2020 Jul 13;24(1):421.

5. Montalvan V, Lee J, Bueso T, De Toledo J, Rivas K. Neurological manifestations of COVID-19 and other coronavirus infections: $A$ systematic review. Clin Neurol Neurosurg. 2020 Jul;194:105921.

6. Wang L, Shen Y, Li M, Chuang H, Ye Y, Zhao H, Wang H. Clinical manifestations and evidence of neurological involvement in 2019 novel coronavirus SARS-CoV-2: a systematic review and meta-analysis. J Neurol. 2020 Oct;267(10):2777-2789.

7. Ganong LH. Integrative reviews of nursing research. Res Nurs Health. 1987;10(1):1-11.

8. Broome ME. Integrative literature reviews for the development of concepts. In: Rodgers BL, Knafl KA, editors. Concept development in nursing: foundations, techniques and applications. Philadelphia (USA): WB Saunders; 2000. p. 231-50.

9. Whittemore R, Knafl K. The integrative review: update methodology. J Adv Nurs. 2005;52(5):546-53

10. Asadi-Pooya AA, Simani L. Central nervous system manifestations of COVID-19: A systematic review. J Neurol Sci. 2020 Jun 15;413:116832.
11. Chen $X$, Laurent $S$, Onur OA, Kleineberg NN, Fink GR, Schweitzer $F$, Warnke $C$. A systematic review of neurological symptoms and complications of COVID-19. J Neurol. 2020 Jul 20:1-11.

12. Romoli M, Jelcic I, Bernard-Valnet R, García Azorín D, Mancinelli L, Akhvlediani T, et al; Infectious Disease Panel of the European Academy of Neurology. A systematic review of neurological manifestations of SARS-CoV-2 infection: the devil is hidden in the details. Eur J Neurol. 2020 Jun 5:10.1111/ene.14382.

13. Pinzon RT, Wijaya VO, Buana RB, Al Jody A, Nunsio PN. Neurologic Characteristics in Coronavirus Disease 2019 (COVID-19): A Systematic Review and Meta-Analysis. Front Neurol. 2020 May 29;11:565.

14. Román GC, Spencer PS, Reis J, Buguet A, Faris MEA, Katrak SM, et al; WFN Environmental Neurology Specialty Group. The neurology of COVID-19 revisited: A proposal from the Environmental Neurology Specialty Group of the World Federation of Neurology to implement international neurological registries. J Neurol Sci. 2020 Jul 15;414:116884.

15. Kremer S, Lersy F, Anheim M, Merdji H, Schenck M, Oesterlé $\mathrm{H}$, et al. Neurologic and neuroimaging findings in patients with COVID-19: A retrospective multicenter study. Neurology. 2020 Sep 29;95(13):e1868-e1882

16. Romero-Sánchez CM, Díaz-Maroto I, Fernández-Díaz E, Sánchez-Larsen Á, Layos-Romero A, García-García J, et al. Neurologic manifestations in hospitalized patients with COVID-19: The ALBACOVID registry. Neurology. 2020 Aug 25;95(8):e1060-e1070

17. Msigwa SS, Wang Y, Li Y, Cheng $X$. The neurological insights of the emerging coronaviruses. J Clin Neurosci. 2020 Aug;78:1-7.

18. Fatima N, Saqqur M, Qamar F, Shaukat S, Shuaib A. Impact of COVID-19 on neurological manifestations: an overview of stroke presentation in pandemic. Neurol Sci. 2020 Oct;41(10):26752679. 